\title{
Arthroscopic Coracoclavicular Ligament Reconstruction of Chronic Acromioclavicular Dislocations Using Autogenous Semitendinosus Graft: A Two-Year Follow-up Study of 58 Patients
}

\author{
Juha O. Ranne, M.D., Ph.D., Terho U. Kainonen, M.D., Janne T. Lehtinen, M.D., Ph.D., \\ Kari J. Kanto, M.D., Heidi A. Vastamäki, M.D., Ph.D., Mari K. Kukkonen, Ph.D., and \\ Mika T. Siitonen, MSc.
}

\begin{abstract}
Purpose: To determine the results of operatively treated chronic acromioclavicular (AC) joint dislocations after 2-year follow-up. Methods: Fifty-eight patients with chronic acromioclavicular separations underwent arthroscopic coracoclavicular ligament reconstructions using semitendinosus autografts. Constant and Simple Shoulder Test scores were determined before and 2 years after surgery as a part of standard clinical practice. General patient satisfaction with the outcome (poor, fair, or excellent) also was assessed. In addition, for purposes of routine clinical follow-up, the coracoclavicular distance was measured from the inferior cortex of the clavicle to the superior cortex of the coracoid using anteroposterior radiographs taken 2 years after surgery. The results were compared with postoperative radiographs and changes in the distance were recorded. The clavicular drill hole was similarly measured 2 years after surgery to detect possible tunnel widening. Results: The mean preoperative Constant score increased from a preoperative mean of $52.6 \pm$ 16.5 to $94.7 \pm 7.9$ at 2 years postoperatively $(P=.000)$. The Simple Shoulder Test score increased from a preoperative mean of $7.7 \pm 1.64$ to $11.8 \pm 0.7(P=.000)$. The mean coracoclavicular distance increased from $10.5 \pm 3.4$ to $12.4 \pm$ $3.9 \mathrm{~mm}(P=.009)$. The diameter of the clavicular drill hole increased from $6.0 \mathrm{~mm}$ to a mean of $8.4 \mathrm{~mm}$. Two coracoid fractures were observed, but no clavicular fractures. One patient experienced a deep infection, leading to total reconstruction failure, and 2 patients had superficial postoperative infections. Forty-five patients $(85 \%)$ reported excellent subjective outcomes, and $8(15 \%)$ reported a fair outcome. Conclusions: The outcomes of this series of coracoclavicular ligament reconstruction were favorable and the number of serious complications was small. However, clavicular wound issues were a significant problem. Coracoclavicular ligament reconstruction is a challenging procedure, but satisfactory results can be achieved with careful patient selection and good technique. Level of Evidence: Level IV, therapeutic case series
\end{abstract}

From the Sports Trauma Research Unit, Hospital Mehiläinen Neo, Turku (J.O.R., T.U.K., H.A.V.); Paavo Nurmi Centre, Department of Physical Activity and Health, the University of Turku, Turku (J.O.R.); Department of Orthopaedics and Traumatology, Tampere University Hospital, Tampere (J.T.L., K.J.K.); Research and Development, Mectalent Oy, Oulu (M.K.K.); and Mectalent Medical Services Oy, Oulu (M.T.S.), Finland.

The authors report that they have no conflicts of interest in the authorship and publication of this article. Full ICMJE author disclosure forms are available for this article online, as supplementary material.

Received June 25, 2019; accepted October 16, 2019.

Address correspondence to Juha O. Ranne, M.D., Ph.D., Hospital Mehiläinen Neo, Joukahaisenkatu 6, 20520 Turku,Finland.E-mail: rannejuha@ gmail.com

(C) 2019 THE AUTHORS. Published by Elsevier Inc. on behalf of the Arthroscopy Association of North America. This is an open access article under the CC BY-NC-ND license (http://creativecommons.org/licenses/by-nc-nd/4.0/). 2666-061X/19463

https://doi.org/10.1016/j.asmr.2019.10.003
A cromioclavicular (AC) joint separation is a comA mon injury, especially among young athletes involved in contact sports. ${ }^{1,2}$ Typical symptoms following trauma are pain and swelling of the AC joint and an elevated distal clavicle position. Shoulder motion also may be reduced due to pain. The typical mechanism of trauma is a direct force on the shoulder girdle driving the acromion inferiorly in relation to the clavicle. This leads to AC joint capsule failure, followed by a disruption of the coracoclavicular (CC) ligaments. In Rockwood type I and II injuries, the capsular ligament ruptures are partial, and the dislocation of the distal clavicle is minor. In type III injuries, the AC and CC ligaments are completely torn, and the distal clavicle appears elevated. In type $\mathrm{V}$ injuries, the force has additionally ruptured the attachments of the 
surrounding muscle insertions on the clavicle. ${ }^{3}$ The scapula moves inferiorly, and the distal clavicle is markedly elevated and unstable. In the rare type IV injury, the distal clavicle is posteriorly displaced into the trapezius muscle, and in type VI injuries, the dislocation is under the coracoid process. ${ }^{4}$

There is general agreement that low-grade AC dislocations (types I-II) should be treated conservatively, whereas high-grade dislocations (types III-VI) may be treated operatively. ${ }^{5,6}$ Conservative treatment typically is offered first for type III separations, and in many cases the distal clavicle stabilizes well. However, in some cases, the distal clavicle remains unstable and painful. The clavicle is the only rigid element that connects the upper extremity to the axial skeleton. An unstable distal clavicle can lead to scapular dyskinesis and SICK (Scapular malposition, Inferior medial border prominence, Coracoid pain and malposition and dysKinesis of scapular movement) scapula syndrome. ${ }^{7-9}$ Therefore, operative treatment also may be needed for type III separations. Operative treatment is generally indicated in type IV, V, and VI injuries. ${ }^{10}$ Athletes and younger active patients often tend to receive operative treatment. ${ }^{11,12}$

The complication rates in surgically treated AC dislocations are comparatively high, with problems varying from hardware failure, implant irritation, and clavicle and coracoid fractures to reconstruction failures and infections. ${ }^{13,14}$ Most of the operative techniques mentioned are still in use today, because as yet, no operative standard has been established for the treatment of this kind of trauma. ${ }^{15,16}$ However, it is possible that rather than one standard technique, several parallel techniques will suffice, depending on the preferences of the surgeon.

The purpose of this study was to determine the results of operatively treated chronic AC joint dislocations after 2 -year follow-up. The hypothesis was that by using a good technique with selected patients, arthroscopic CC ligament reconstruction would be associated with favorable clinical and radiographic outcomes, although the procedure has a risk for clavicular wound infection and a coracoid or clavicular fracture.

\section{Methods}

In this series, patients with Rockwood grade III and V chronic AC separations were treated using the surgical technique described by Ranne et al. between 2009 and 2016. ${ }^{17,18}$ The patients were followed for 2 years after surgery. All procedures except one (a revision case after previous surgery failed) were primary operations. The patients were carefully selected before surgery, and the included patients were individuals motivated to strictly follow the instructions given after surgery. The exclusion criteria in this study were excessive additional trauma such as rotator cuff tears requiring repair and distal clavicle fractures. All patients were operated on using the same arthroscopic technique by senior surgeons. The Constant score and the Simple Shoulder Test were obtained both before and 2 years after surgery. General patient satisfaction with the outcome (poor, fair, or excellent) was assessed 2 years after surgery. Using anteroposterior radiographs taken postoperatively and 2 years after surgery, the CC distance was measured from the inferior cortex of the clavicle to the superior cortex of the coracoid recording any change in the distance. The measurements were made by the surgeons using a scale of the imaging program. Similarly, the clavicular drill hole also was measured to detect any possible tunnel widening. The indications for surgical treatment in this study included AC joint pain, distal clavicle instability, and scapular problems. Treatment selection was not based on the radiographic grading of the AC separation alone but on the discomfort and pain experienced by the patient.

Statistical analyses were calculated using Minitab software, version 18.1 (State College, PA). This includes descriptive univariate statistics (arithmetic mean, standard deviation), 95\% confidence intervals, $t$ tests, and graphs. Welch's $t$ test was used to compare whether preoperative and postoperative groups differ from each other. For CC distance, Welch's $t$ test result was verified with classic 2-sample $t$ test, as variances of these populations were similar. For all $t$ tests, the null hypothesis stated that the difference between pre- and postoperative groups is 0 . Because all the $P$ values were less than the significance level, null hypothesis was rejected and it could be concluded that there is a difference between means of pre- and postpopulations. For clavicle drill hole diameter, significance could not be calculated, as perioperative diameter was not measured but nominal drill diameter was used instead. However, in this case, postoperative $95 \%$ confidence interval of mean was visually compared with the perioperative drill diameter to verify the statistically significant increase in drill-hole diameter. In other variables than drill hole diameter, statistical significance was declared when $P<.05$. The study was approved by the institutional research board where the research was conducted (Hospital Mehiläinen Neo, Turku, Finland).

\section{Surgical Technique}

The key element of the technique is the positioning of the semitendinosus graft. The technique involves a double-bundle reconstruction of the CC ligament complex using the tendon graft. The anterior graft limb projects superiorly and replaces the trapezoid ligament while the dorsal limb of the graft is wrapped around the dorsal edge of the clavicle, reconstructing the conoid ligament (Fig 1). This effectively stabilizes the AC joint and reduces the anteroposterior translation of the distal clavicle. A supportive semitemporary fixation of the 


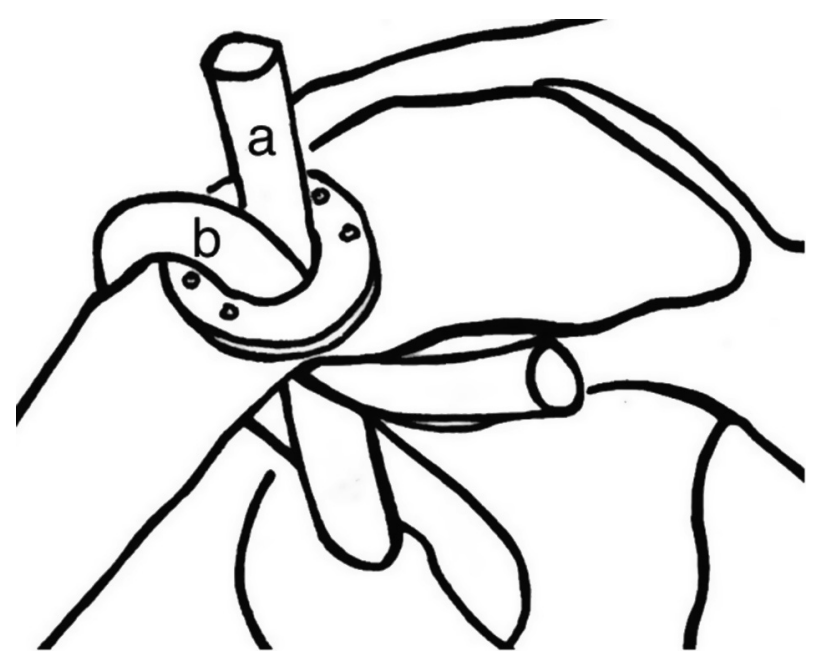

Fig 1. The anterior tendon graft limb is fixed to the middle of the clavicle, recreating the trapezoid ligament (a). The posterior graft limb is wrapped around the posterior edge of the clavicle, recreating the conoid ligament (b). Left shoulder, anteromedial view.

graft reconstruction was achieved by connecting a GraftWasher button (Arthrex, Naples, FL) on the clavicle to a subcoracoid button with a strong no. 5 nonabsorbable suture or tape (Fig 2). As a subcoracoid counterpart the DogBone button (Arthrex) proved to be a practical solution. The semitendinosus tendon graft shared the same drill holes with the supportive fixation, and because there was only one centrally positioned 6-mm drill hole on the clavicle, the risk for a fracture was minimal. The patient was placed in the beach chair position to harvest the ipsilateral semitendinosus tendon using a special instrument for the purpose and its thickness was measured. The semitemporary fixation device was assembled and readied for the operation.

Arthroscopy was initiated by inserting the arthroscope into the joint through the posterior portal, and the joint was checked for possible additional pathology. The lateral portal was opened by inserting a needle in the front of the long-head biceps tendon aimed at the proximal coracoid. The coracoid neck was exposed using an electrocautery. The primary camera position during the actual reconstruction was in the lateral portal. The anterolateral portal was established by using a needle pointing to the coracoid neck. The distal clavicle was released arthroscopically to ensure tensionfree as repositioning but was not routinely resected. The $2.5-\mathrm{cm}$ sagittal clavicular portal or wound was opened $2.5 \mathrm{~cm}$ proximal to the AC joint. The exact position of the portal was established using a needle. The needle was positioned approximately $1 \mathrm{~cm}$ posterior to the dorsal edge of the clavicle to easily reach the coracoid neck area. The superior surface of the clavicle was exposed for drilling through the same opening.
Conventional drill guides were used. First, a 4.5-mm hole was drilled through the clavicle and coracoid using a guide pin. As the graft was to be fixed to the clavicular tunnel with a $5.5-\mathrm{mm} \times 10-\mathrm{mm}$ PEEK (polyether ether ketone) interference screw, the clavicular drill hole was enlarged to $6 \mathrm{~mm}$. The passing sutures for both the graft and fixation apparatus were pulled through the bone tunnels using the lasso technique. The graft was first pulled through the clavicular and coracoid drill holes to the anterolateral portal. The graft end was then pulled to the clavicular portal behind the clavicle. The interconnecting suture was then pulled through the drill holes beside the graft to the anterolateral portal. The subcoracoid button was attached to the suture loop and pulled back in to its placement underneath the coracoid by the superior suture ends. The end of the anterior limb was slipped straight through the clavicular washer whereas the end of the dorsal limb was extended over the dorsal rim of the washer and then slipped through the clavicular drill hole adjacent to the anterior graft limb. It was then drawn to the anterolateral portal using the lasso technique.

At this point, with the entire length of the graft and the fixation apparatus in place, the repositioning of the AC joint was checked arthroscopically to see that the clavicle and acromion were at the same level. With the repositioning reliably completed, the fixation device was tightened and the graft tensioned simultaneously.

The arm was held in an arm holder in a slight anterior flexion. The anterior graft limb was tensioned by directly pulling superiorly while the posterior graft limb was tensioned by pulling horizontally from the anterolateral portal. The suture ends were tied over the washer while maintaining the tension of the graft. The graft was then fixed to the clavicular tunnel with a 5.5-mm $\times 10-\mathrm{mm}$ PEEK interference screw. Finally, the anterior graft limb was sutured on to the dorsal graft limb behind the clavicle using unresorbable no. 2 suture, and the excess graft ends were removed. The arthroscopic portals were closed with interrupted sutures, whereas the clavicular wound was closed in layers to cover the washer and protruding suture knots. The patients were discharged the same day and wore an arm sling for 4 weeks. They were allowed for light rotatory movements and passive arm lifting within the limits of pain. Although the sling was removed after 4 weeks, active rehabilitation did not begin until 8 weeks after surgery to allow enough time for recovery. The patients were allowed to resume heavy labor 3 to 4 months after surgery and overhead activity and sports at 6 months.

\section{Results}

The results for 58 patients with surgically treated chronic type III and V AC separations (29 of each) are 

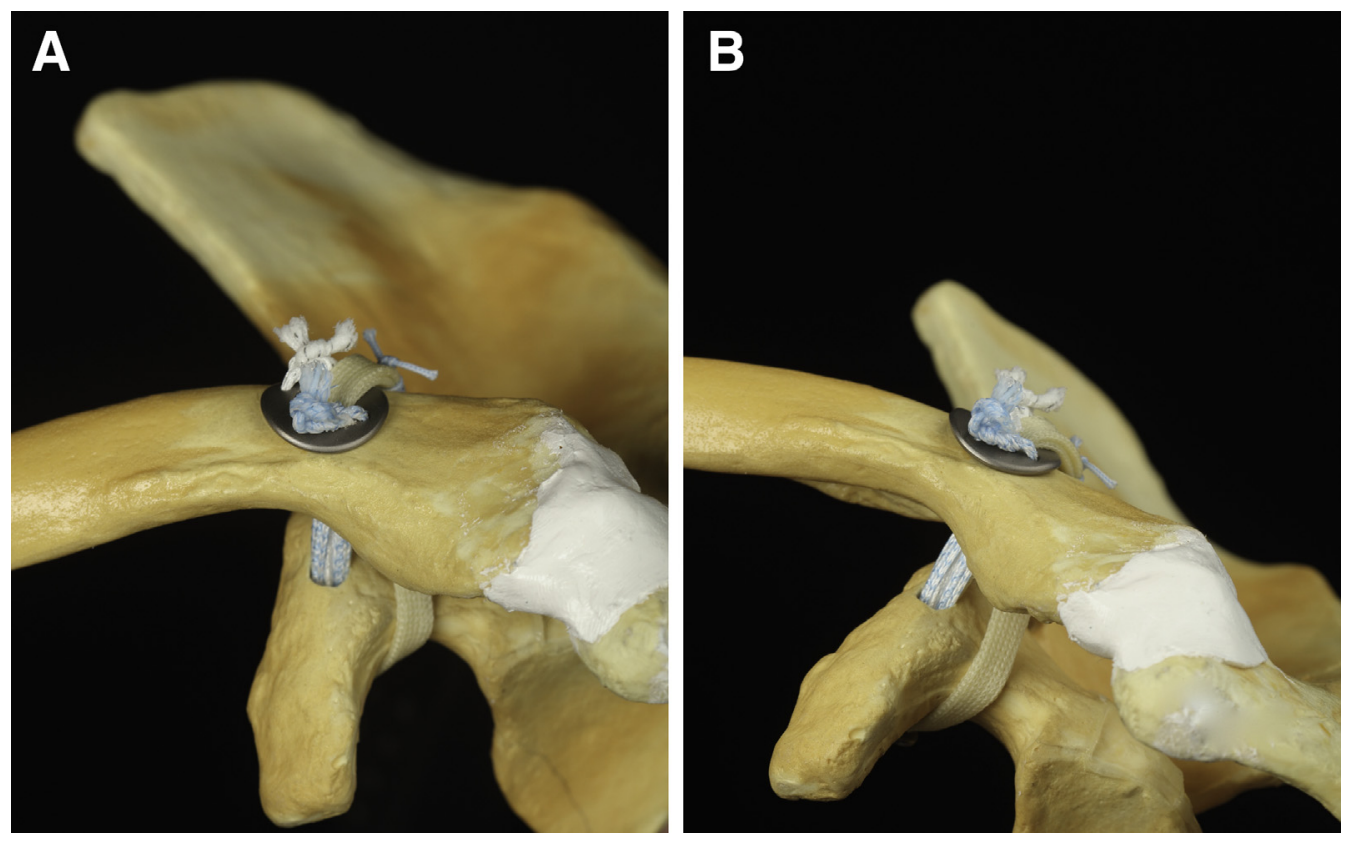

Fig 2. (a) Anterior view of the complete reconstruction of a left shoulder. The semitendinosus graft and the interconnecting sutures share the same drill holes. (b) Anterolateral view of the complete reconstruction.

reported in this series. The time from trauma to surgery varied from 3 weeks to 10 years. Fifty-four cases were male, 4 were female, and the mean age of the patients was $36.38 \pm 13.30$ years. Thirty-two patients $(55 \%)$ had injured their dominant side shoulder. Five patients were lost from the follow-up: 1 case of deep infection that led to graft removal; 1 case of subsequent, unrelated severe external trauma to the operated shoulder; and 3 cases that could not reached. One noncompliant patient was excluded from the follow-up.

The remaining 53 patients were able to return to sports and heavy labor. The mean Constant score increased from a preoperative mean $52.6 \pm 16.5$ to 94.7 \pm 7.9 at 2 years postoperatively $(P=.000)$ (Fig 3$)$. The Simple Shoulder Test score increased from a preoperative mean $7.8 \pm 1.6$ to $11.8 \pm 0.7(P=.000)$ (Fig 4$)$. The CC distance increased from $10.5 \pm 3.4$ to $12.4 \pm 3.9$ $(P=.009)$ (Fig 5). The clavicular drill hole diameter increased from $6 \mathrm{~mm}$ to a mean of $8.4 \pm 1.2 \mathrm{~mm}$ (Figs 6 and 7). There were no clavicular fractures. There were 2 cases of coracoid fractures in this study, although in both cases the treatment was nonoperative. In another case (an ice hockey player), a coracoid fracture was detected during a routine radiograph 2 years after the operation, but the patient had no clinical symptoms, and the AC joint was stable. One patient had a deep infection, and 2 had superficial wound infections. In 2 cases, the irritating suture knots on the clavicle were removed in a later phase. Patient satisfaction was very high. Among the 53 patients, $45(85 \%)$ reported an excellent outcome, and $8(15 \%)$ reported a fair outcome.

\section{Discussion}

The AC joint is a synovial joint that connects the acromion to the distal clavicle. Medially, the clavicle is connected to the sternum by the sternoclavicular joint. The distal clavicle is stabilized by the CC ligament complex and the AC joint capsule. ${ }^{19}$ The CC ligament complex connects the clavicle and the coracoid process and provides the AC joint with vertical stability, primarily. The CC ligament complex consists of the lateral trapezoid and medial conoid ligaments, which originate from the superior surface of the coracoid process. The AC joint capsule includes 4 ligamentous thickenings (anterior, posterior, superior, and inferior ligaments), which provide anteroposterior stability to the AC joint. ${ }^{20-23}$

Historically, open procedures have been the dominant surgical treatment strategy, but minimally invasive techniques have been developed since the introduction of arthroscopic surgery. The original techniques included a temporary fixation of the distal clavicle with CC screws, tension band fixations, and hook plates, assuming that the scar tissue would fasten the clavicle to its surroundings. ${ }^{24-26}$ With the exception of the Weaver-Dunn technique, where the coracoacromial ligament is used as a reconstructive graft, tendon grafts are usually not used in these techniques. ${ }^{27}$ Many of the current techniques include CC ligament reconstruction 
Constant score

$95 \% \mathrm{Cl}$ for the Mean

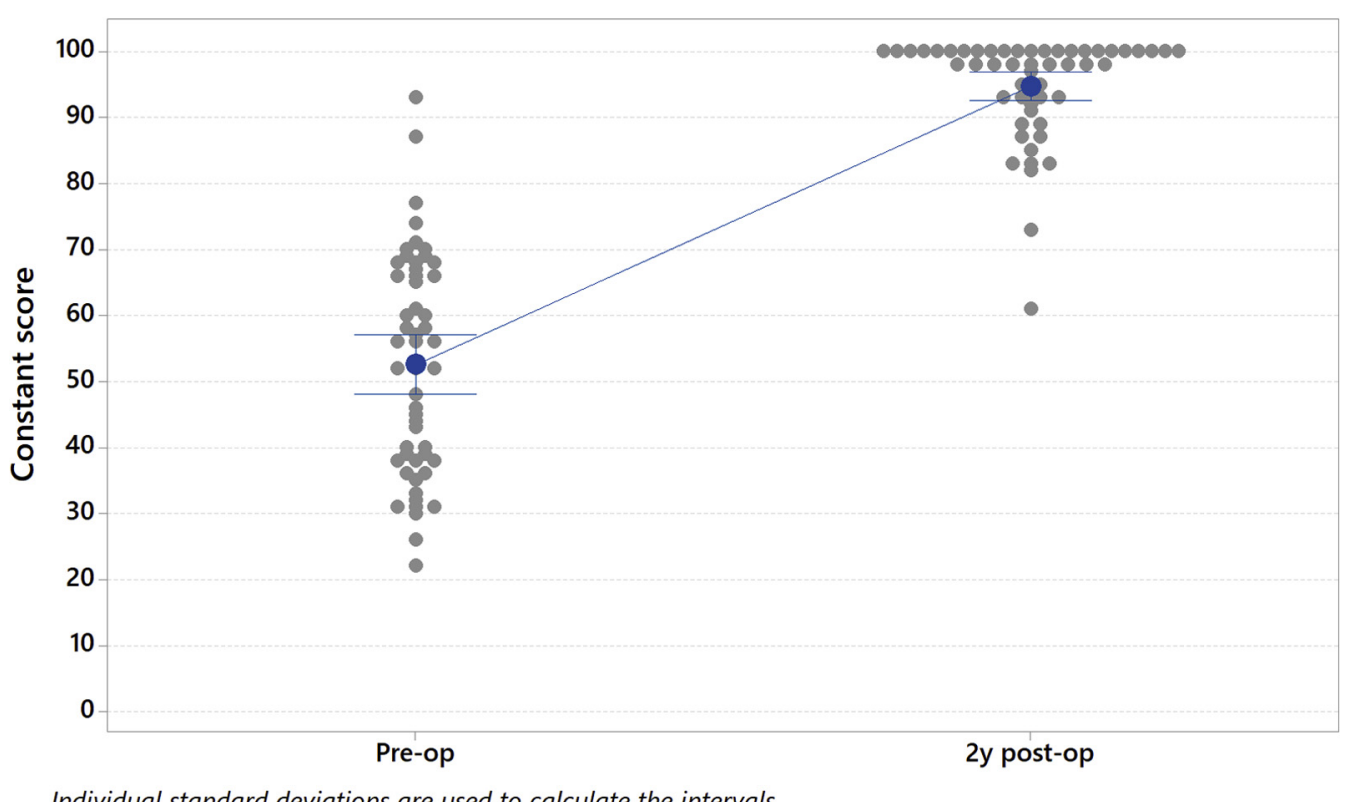

Individual standard deviations are used to calculate the intervals.

Fig 3. Constant score populations with individual values and $95 \%$ CIs. The mean preoperative constant score increased 42.15 points from $52.57 \pm 16.46$ to $94.72 \pm 7.89$ at 2 years postoperatively $(P=.000)$. Constant score ranged from 0 to 100 points. $(\mathrm{CI}$, confidence interval.)

using a hamstring tendon graft in an attempt to achieve an anatomic solution and enhance the results' longevity, especially in chronic cases. ${ }^{28-33}$ Although hamstring tendon autografts and allografts are the most commonly used in surgical procedures, new techniques using artificial tendon grafts also have emerged. ${ }^{34-36}$ In addition, many current techniques do not use a tendon graft and instead rely on suture slings, especially in

\section{Simple shoulder test (SST) score $95 \% \mathrm{Cl}$ for the Mean}

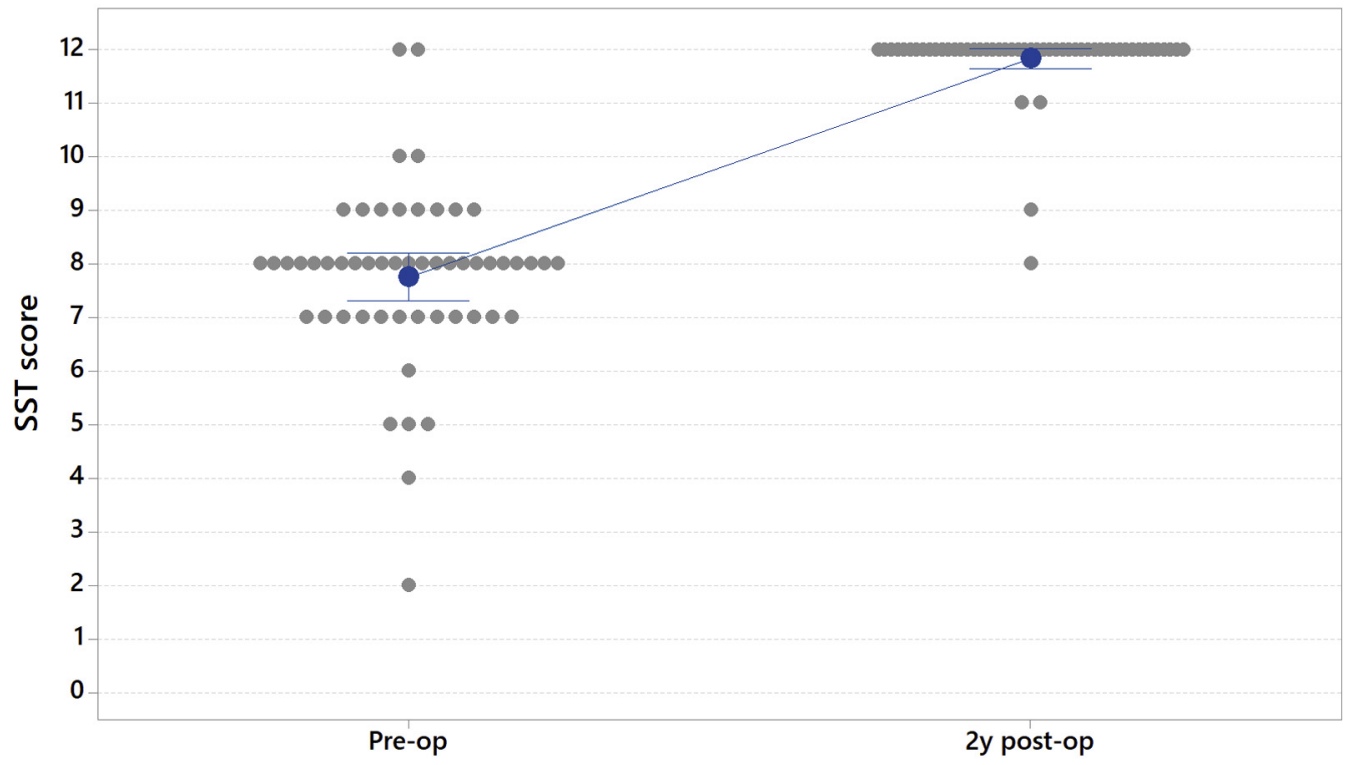

Individual standard deviations are used to calculate the intervals.

Fig 4. Simple Shoulder Test with individual values and $95 \%$ CIs. The mean preoperative simple shoulder test score increased 4.08 points from $7.75 \pm 1.64$ to $11.83 \pm 0.70$ at 2 years postoperatively $(P=.000)$. Simple Shoulder Test score ranged from 0 to 12 points. (CI, confidence interval.) 


\section{Coraco-clavicular distance (RTG) $95 \% \mathrm{Cl}$ for the Mean}

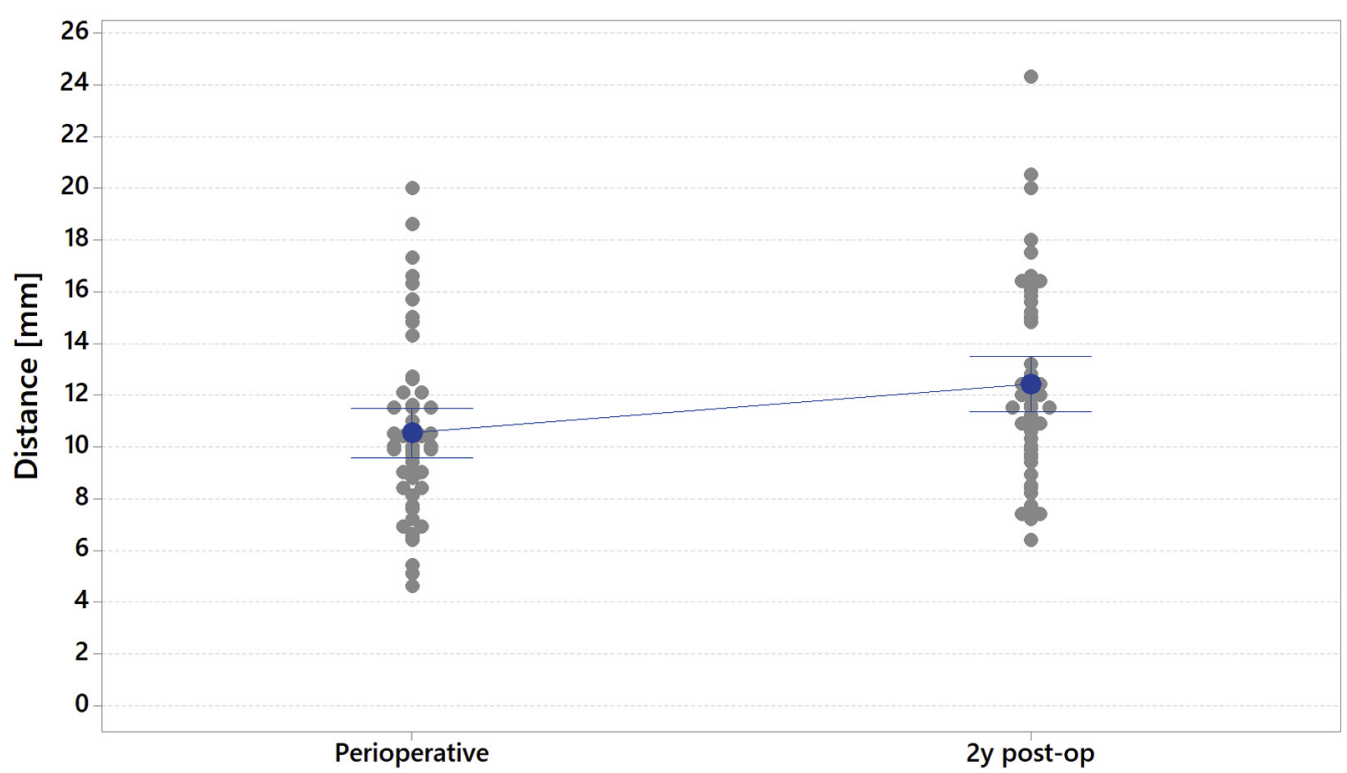

Individual standard deviations are used to calculate the intervals.

Fig 5. Coracoclavicular distance with individual values and $95 \%$ confidence intervals. The mean coracoclavicular distance increased $1.89 \mathrm{~mm}$ from $10.54 \pm 3.41 \mathrm{~mm}$ to $12.43 \pm 3.86 \mathrm{~mm} 2$ years postoperatively $(P=.009)$. (CI, confidence interval; RTG, anteroposterior radiograph.)

acute cases. ${ }^{37-39}$ However, the techniques that reconstruct the CC ligament complex with a tendon graft are significantly better than suture sling methods at stabilizing anteroposterior translation. ${ }^{31}$ The AC joint capsule also plays an important role in the distal clavicle's anteroposterior stability, and addressing AC joint capsule failure can enhance this stability. ${ }^{30,40,41}$

The Constant and Simple Shoulder Test scores were significantly improved in this follow-up study, and general patient satisfaction was high. These outcome

\section{Clavicle drill hole diameter (RTG) $95 \% \mathrm{Cl}$ for the Mean}

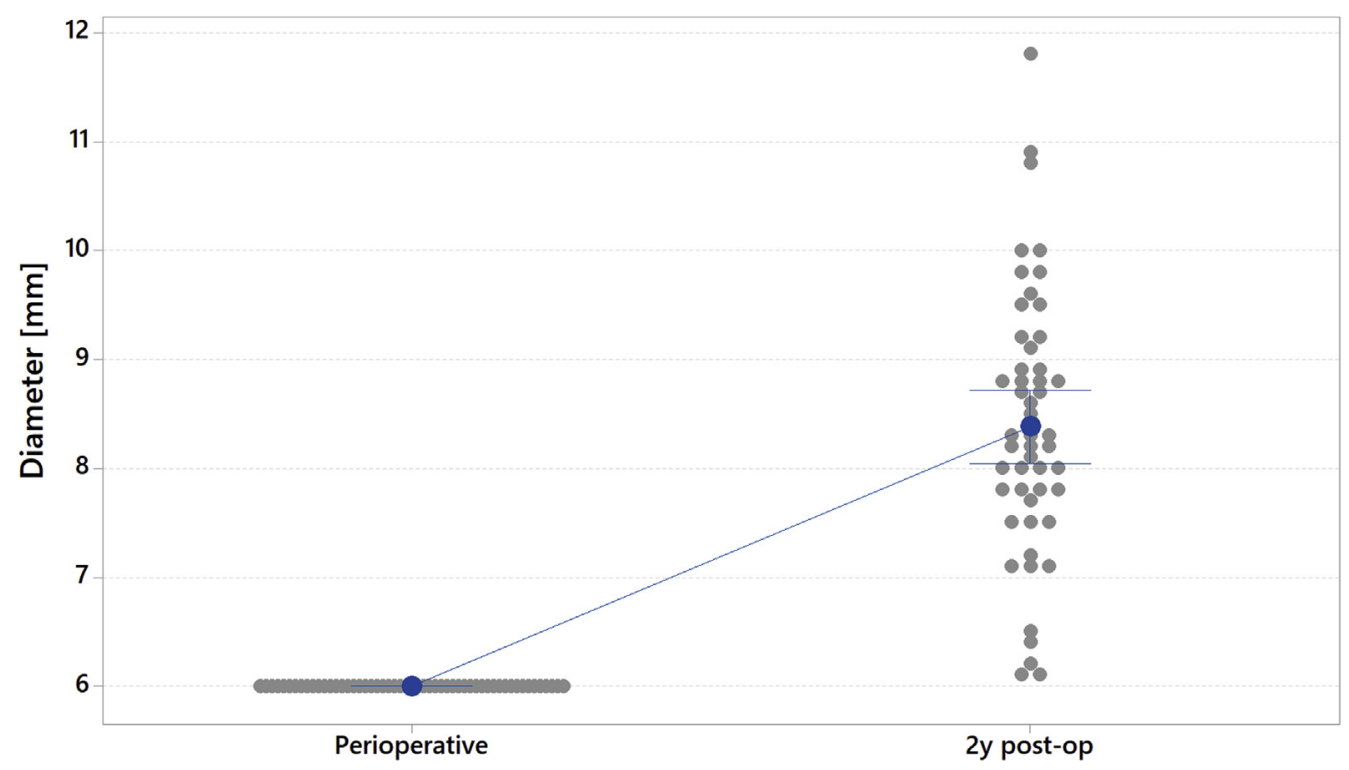

Fig 6. The diameter of the clavicular drill hole increased on an average $2.39 \mathrm{~mm}$ from $6 \mathrm{~mm}$ to mean $8.39 \pm 1.22 \mathrm{~mm}$. (CI, confidence interval; RTG, anteroposterior radiograph.)

Individual standard deviations are used to calculate the intervals. 


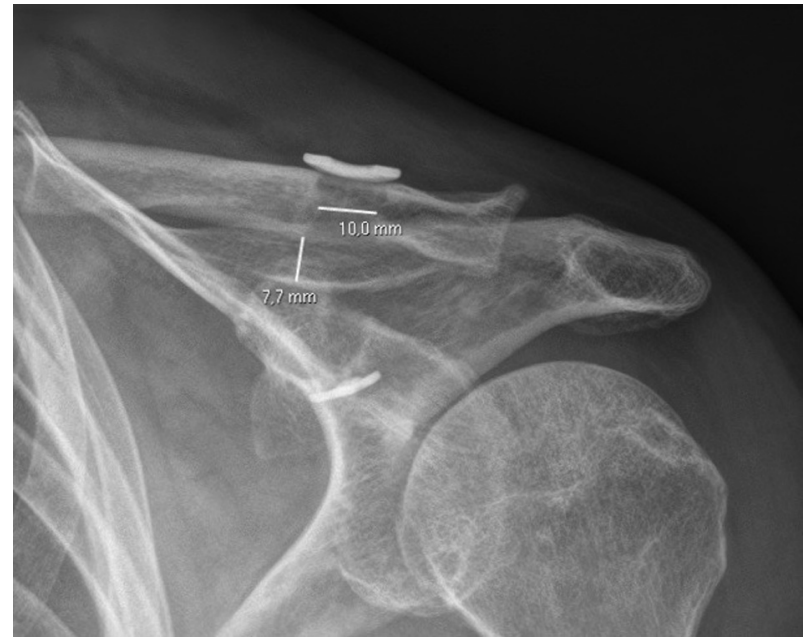

Fig 7. Two-year postoperative anteroposterior radiograph of a left shoulder with the measurement markings for the coracoclavicular distance $(7.7 \mathrm{~mm})$ and the clavicular drill hole (10.0 mm).

measures were used as practical tests and indicate the functional and subjective condition of the shoulder. Patient satisfaction was closely related to the score outcomes. The AC joint-specific outcome score, the Nottingham Clavicle Score, was not used because this study was performed before it was published. ${ }^{42}$

In this technique, a 6-mm clavicular drill hole was required to house the graft that was pulled through the bone tunnels twice and using a 5.5-mm interference screw. Tunnel widening was detected during the follow-up, but if centrally positioned, the single anatomically positioned drill hole does not carry a risk of clavicular fracture. ${ }^{43}$ The tendon graft, interconnecting sutures, and interference screw are the probable reasons for tunnel widening. A $4.5-\mathrm{mm}$ drill hole was required for the coracoid process to pass the semitendinosus graft, and this does carry a risk for the coracoid fracture if improperly positioned. ${ }^{44}$ There was 1 patient with a deep infection and to restrain it, the implants and entire reconstruction had to be removed, leading to reconstruction failure. Two patients suffered a superficial infection of the clavicular wound. The most common complaint made by the patients was related to the suture knots, which were easily palpable on the clavicle. These irritating knots were removed in 2 patients under local anesthesia. However, new techniques have been developed to address the difficulties associated with suture knots. ${ }^{32,41}$

The authors currently use a modified technique using a clavicular implant that hides the interconnecting suture knot in the clavicular drill hole. This technique does not require an interference screw and therefore reduces the amount of foreign material underneath the clavicular wound. ${ }^{41}$ The semitendinosus graft also can be wrapped around the coracoid, avoiding the $4.5-\mathrm{mm}$ drill hole and thus minimizing the risk for a coracoid fracture. After finishing the arthroscopic CC reconstruction, we extend the clavicular wound over the AC joint. The AC capsule is opened along its fibers and the distal end of the clavicle is carefully mobilized and reduced. After that, the CC reconstruction is tensioned. Finally, the AC capsule is plicated and sutured tight with interrupted no. 1 sutures to further stabilize the anteroposterior translation of the distal clavicle. The outcomes of this modified technique have been promising.

\section{Limitations}

Only motivated, compliant patients were included in this study. Inclusion of noncompliant patients (with restrictions of rehabilitation) could result in inferior outcomes. The procedures also were conducted by experienced surgeons who routinely use the technique. That may have an effect to the outcomes in general. Lastly, various outcome scores have been used in previous studies, and therefore, these results cannot be directly compared with those of other studies.

\section{Conclusions}

The outcomes of this series of CC ligament reconstruction were favorable, and the number of serious complications was small. However, clavicular wound issues were a significant problem. CC ligament reconstruction is a challenging procedure, but satisfactory results can be achieved with careful patient selection and good technique.

\section{References}

1. Chillemi C, Franceschini V, Dei Giudici L, et al. Epidemiology of isolated acromioclavicular joint dislocation. Emerg Med Int 2013:171609.

2. Pallis M, Cameron KL, Svoboda SJ, Owens BD. Epidemiology of acromioclavicular joint injury in young athletes. Am J Sports Med 2012;40:2072-2077.

3. Lee S, Bedi A. Shoulder acromioclavicular joint reconstruction options and outcomes. Cur Rev Musculoskelet Med 2016;9:368-377.

4. Rockwood CA. Disorders of the acromioclavicular joint. In: Rockwood CA, Matsen FA, eds. The shoulder. Ed 4. Philadelphia: WB Saunders, 2009;453-526.

5. Beitzel K, Cote MP, Apostolakos J, et al. Current concepts in the treatment of acromioclavicular joint dislocations. Arthroscopy 2013;29:387-397.

6. Beitzel K, Mazzocca AD, Bak K, et al. ISAKOS upper extremity committee consensus statement on the need for diversification of the Rockwood classification for acromioclavicular joint injuries. Arthroscopy 2014;30:271-278.

7. Gumina S, Carbone S, Postacchini F. Scapular dyskinesis and SICK scapula syndrome in patients with chronic type III acromioclavicular dislocation. Arthroscopy 2009;25:40-45.

8. Kibler WB, Ludewig PM, McLure P, Michener LA, Bak K, Sciascia A. Clinical implications of scapular dyskinesis in 
shoulder injury: The 2013 consensus statement from the 'scapular summit'. Br J Sports Med 2013;47:877-885.

9. Murena L, Canton G, Vulcano E, Cherubino P. Scapular dyskinesis and SICK scapula syndrome following surgical treatment of type III acute acromioclavicular dislocations. Knee Surg Sports Traumatol Arthrosc 2013;21:1146-1150.

10. Modi CS, Beazley J, Zywiel MG, Lawrence TM, Veillette CJ. Controversies relating to the management of acromioclavicular joint dislocations. Bone Joint J 2013;95: 1595-1602.

11. Tamaoki MJS, Belloti JC, Lenza M, Matsumoto MH, Dos Santos G, Faloppa F. Surgical versus conservative interventions for treating acromioclavicular dislocation of the shoulder in adults. Cochrane Database Syst Rev 2010;4: CD007429.

12. Trainer G, Arciero RA, Mazzocca AD. Practical management of grade III acromioclavicular separations. Clin J Sports Med 2008;18:162-166.

13. Gowd AK, Liu JN, Cabarcas BC, et al. A systematic review and meta-analysis of operative techniques. Am J Sports 2019;47:2745-2758.

14. Frank RM, Cotter EJ, Leroux TS, Romeo AA. Acromioclavicular joint injuries: Evidence-based treatment. J Am Acad Orthop Surg 2019;27:e775-e788.

15. Brand JC, Lubowitz JH, Provencher MT, Rossi MJ. Acromioclavicular joint reconstruction: Complications and innovations. Arthroscopy 2015;31:795-797.

16. Millett PJ, Horan MP, Warth RJ. Two-year outcome after primary anatomic coracoclavicular ligament reconstruction. Arthroscopy 2015;31:1962-1973.

17. Ranne JO, Sarimo JJ, Rawlins MI, Heinonen OJ, Orava SY. All-arthroscopic double-bundle coracoclavicular ligament reconstruction using autogenous semitendinosus graft: A new technique. Arthroscopy Tech 2012;1:el1-el4.

18. Weinstein DM, McCann PD, McIlveen SJ, Flatow EL, Bigliani LU. Surgical treatment of complete acromioclavicular dislocations. Am J Sports Med 1995;23:324-331.

19. Renfree KJ, Wright TW. Anatomy and biomechanics of the acromioclavicular and sternoclavicular joints. Clin Sports Med 2003;22:219-237.

20. Fukuda K, Craig EV, An K, Cofield RH, Chao EY. Biomechanical study of the ligamentous system of the acromioclavicular joint. J Bone Joint Surg 1986;68A: 434-440.

21. Klimikiewicz JJ, Williams GR, Sher JS, et al. The acromioclavicular capsule as a restraint to posterior translation of the clavicle: A biomechanical analysis. J Shoulder Elbow Surg 1999;8:119-124.

22. Lee KW, Debski RE, Chen C-H, et al. Functional evaluation of the ligaments at the acromioclavicular joint during anteroposterior and superoinferior translation. Am J Sports Surg 1997;25:858-862.

23. Terry GC, Chopp TM. Functional anatomy of the shoulder. J Athl Train 2000;35:248-255.

24. Bosworth BM. Acromioclavicular separation: New method for repair. Surg Gynecol Obstet 1941;73:866-871.

25. Lee YS, Lau MJ, Tseng YC, Chen WC, Kao HY, Wei JD. Comparison of the efficacy of hook plate versus tension band wire in the treatment of unstable fractures of the distal clavicle. Int Orthop 2009;33:1401-1405.
26. Moatshe G, Kruckeberg BM, Chahla J, et al. Acromioclavicular and coracoclavicular ligament reconstruction for acromioclavicvular joint instability: A systematic review of clinical and radiographic outcomes. Arthroscopy 2019;34:1979-1995.

27. Weaver JK, Dunn HK. Treatment of acromioclavicular injuries, especially complete acromioclavicular separation. J Bone Joint Surg Am 1972;54:1187-1194.

28. Carofino BC, Mazzocca AD. The anatomic coracoclavicular ligament reconstruction: Surgical technique and indications. J Shoulder Elbow Surg 2010;19:37-46.

29. Lee SJ, Nicholas SJ, Akizuki KH, McHugh MP, Kremenic IJ, Ben Avi S. Reconstruction of the coracoclavicular ligaments with tendon grafts. A comparative biomechanical study. Am J Sports Med 2003;31:648-655.

30. Martetshläger F, Tauber M, Habermeyer P, Hawi N. Arthroscopically assisted acromioclavicular and coracoclavicular ligament reconstruction for chronic acromioclavicular joint instability. Arthrosc Tech 2016;5: el239-el246.

31. Mazzocca AD, Santangelo SA, Johnson ST, Rios CG, Dumonski ML, Arciero RA. A biomechanical evaluation of an anatomical coracoclavicular ligament reconstruction. Am J Sports Med 2006;34:236-246.

32. Menge TJ, Tahal DS, Katthagen JG, Millett PJ. Arthroscopic acromioclavicular joint reconstruction using knotless coracoclavicular ligament reconstruction. Arthrosc Tech 2017;6:e37-e42.

33. Millett PJ, Warth RJ, Greenspoon JA, Horan MP. Arthroscopically assisted anatomic coracoclavicular ligament reconstruction technique using coracoclavicular fixation and soft-tissue grafts. Arthrosc Tech 2015;4: e583-e587.

34. Marcheggiani Muccioli GM, Manning C, Wright P, Grassi A, Zaffagnini S, Funk L. Acromioclavicular joint reconstruction with the LARS ligament in professional versus nonprofessional athletes. Knee Surg Sports Traumatol Arthrosc 2016;24:1961-1967.

35. Ranne JO, Kainonen TU, Lempainen LL, Kosola JA, Kajander SA, Niemi PT. Arthroscopic coracoclavicular ligament reconstruction using a synthetic polycaprolactone-based polyurethane urea tendon graft: A report of 5 cases. Surg Innov 2018;25:224-229.

36. Triantafyllopoulos IK, Lampropoulou-Adamidou K, Shizas NP, Karadimas EV. Surgical treatment of acute type $\mathrm{V}$ acromioclavicular joint dislocations in professional athletes: An anatomic ligament reconstruction with synthetic implant augmentation. J Shoulder Elbow Surg 2017;26:e369-e375.

37. Haber DB, Golijanin P, Stone GL, et al. Primary acromioclavicular-coracoclavicular reconstruction using 2 allografts, TightRope and stabilization to the acromion. Arthrosc Tech 2019;8:s147-152.

38. Brock J, Lee PY, Woodford C, Robertson A. A radiological and clinical evaluation of acromioclavicular joint reconstruction using Dog Bone double endobutton technique. $J$ Arthritis 2016;5:1-4.

39. Pan X, Zhang H, Sun C, et al. Arthroscopy-assisted reconstruction of coracoclavicular ligament by endobutton fixation for treatment of acromioclavicular joint dislocation. Arch Orthop Trauma Surg 2015;135:9-16. 
40. Pühringer N, Agneskirchner J. Arthroscopic technique for stabilization of chronic acromioclavicular joint instability with coracoclavicular and acromioclavicular ligament reconstruction using gracilis tendon graft. Arthrosc Tech 2017;6:e175-e181.

41. Ranne J, Kainonen TU, Kosola JA, et al. Arthroscopic coracoclavicular ligament reconstruction using graft augmentation and titanium implants. Arthrosc Tech 2018;7:e465-471.

42. Charles ER, Kumar V, Blacknall J, et al. A validation of the Nottingham clavicle score: A clavicle, acromioclavicular joint and sternoclavicular joint specific patient- reported outcome measure. J Shoulder Elbow Surg 2017;26:1732-1739.

43. Dumont GD, Russell RD, Knight JR, et al. Impact of tunnels and tenodesis screws on clavicle fracture: A biomechanical study of varying coracoclavicular ligament reconstruction techniques. Arthroscopy 2013;29: 1604-1607.

44. Ferreira JV, Chowaniec D, Obopilwe E, Nowak MD, Arciero RA, Mazzocca AD. Biomechanical evaluation of effect of coracoid tunnel placement on load to failure of fixation during repair of acromioclavicular joint dislocations. Arthroscopy 2012;28:1230-1236. 